\title{
Differential Uptake and Trafficking of Nanoparticles by Living Cells
}

\author{
J.A. Timlin, $*$ J.S. Aaron,* Adrienne Greene**, and G.D. Bachand** \\ * Biofuels \& Biodefense Technologies, Sandia National Laboratories, PO Box 5800, Albuquerque, \\ NM 87185-0895 \\ ** Center for Integrated Nanotechnologies, Sandia National Laboratories, Albuquerque, NM 87185 - \\ 1315
}

Engineered nanoparticles are becoming increasingly commonplace in commercial products as well as biomedical research settings. Surprisingly, however, systematic studies of the health hazards of engineered nanomaterials have severely lagged behind their development and application. It has been shown in some cases that nanomaterials can elicit unique and potentially deleterious physiological responses that are not observed with bulk materials of the same type [1].

As part of a larger effort to assess the toxicology of engineered nanoparticles, we have employed advanced microscopy and analysis methods to study the interaction of semiconductor quantum dots (QDs) with living cells. QDs have shown utility as optical biological tracers due to their increased fluorescence quantum yield and enhanced photostability over traditional organic dyes. These characteristics also make them suitable for investigation with optical microscopy. We have employed total internal reflectance fluorescence (TIRF) microscopy [2] to study the behavior of QDs on the cell surface. This method allows us to selectively excite only those nanoparticles on or within the cell membrane, and permits us to study (1) membrane diffusion characteristics of QDs as a function of size and surface properties using single particle tracking, as well as (2) their internalization rate by monitoring the decrease in signal over time as QDs proceed into the cytosol.

In addition we have employed hyperspectral confocal fluorescence microscopy to study the distribution of QDs after internalization. Hyperspectral confocal fluorescence microscopy acquires a fluorescence emission spectrum from each voxel within the field of view [3]. Because the peak of the fluorescence emission spectrum of a QD is correlated with the size of the QD, hyperspectral confocal fluorescence microscopy permits visualization several different quantum dot sizes simultaneously inside a living cell with high spatial resolution.

Using these advanced imaging methods we have investigated - $\mathrm{COOH}$ surface functionalized $\mathrm{CdSe}$ QDs of three sizes (corresponding to emission peaks of $585 \mathrm{~nm}, 605 \mathrm{~nm}$, and $655 \mathrm{~nm}$ ) interacting with rat mast cells. Single QD tracking on the cell surface reveals a range spanning $>10$ orders of magnitude in effective 2D diffusion constants, indicating a highly complex, heterogeneous environment. Further, QD internalization rates were found to be on the order of 5 minutes in cultured RBL cells, as seen in Figure 1A, although there is a non-linear relationship between membrane-residence time and particle size (Figure 1B). Interestingly, results indicate greater internalization and sequestration into lysosomes for both the smallest and largest particle sizes, but not the intermediate size studied, corresponding with the measured membrane residence time.

The combination of imaging modalities enables quantitative measurements of membrane and subcellular interactions of nanoparticles in living cells, leading to a greater mechanistic understanding of nanomaterial interactions in biological systems [4]. 
References

[1] N. Lewisnki et al., Small 4 (2008) 28.

[2] D. Axelrod, Traffic. 2 (2001) 764.

[3] M.B. Sinclair et al., Appl. Optics 45 (2006) 6283.

[4] Sandia is a multiprogram laboratory operated by Sandia Corporation, a Lockheed Martin Company, for the United States Department of Energy's National Nuclear Security Administration under contract DE-AC04-94AL85000. This research was supported by Sandia's Laboratory Directed Research and Development Program.
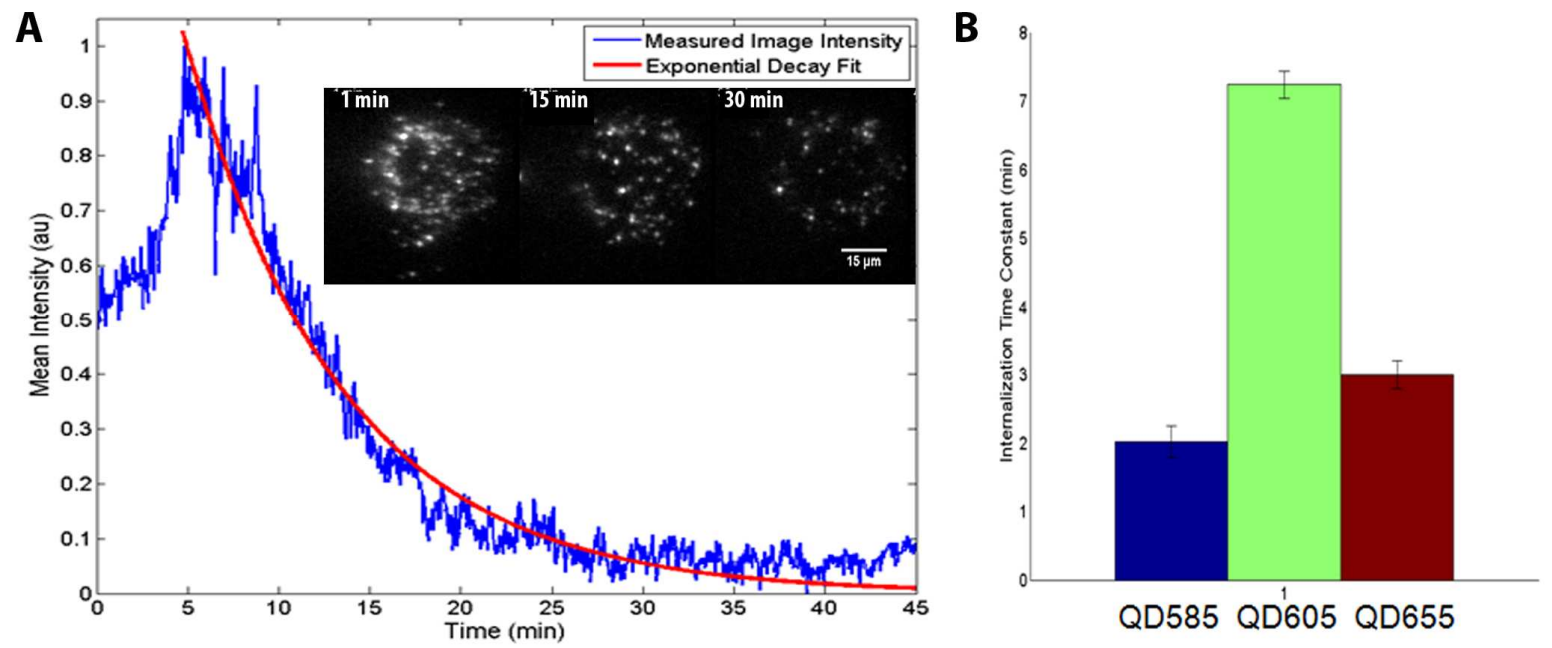

FIG. 1. TIRF microscopy provides an accurate method for measuring membrane interactions of QDs. In (A), QDs are seen diffusing through the membrane, but disappear over 30 min as particles are internalized by the cell. The apparent decrease in signal is modeled as an exponential decay, thus giving a characteristic membrane-residence time for each QD type, which did not vary linearly with particle size (B).
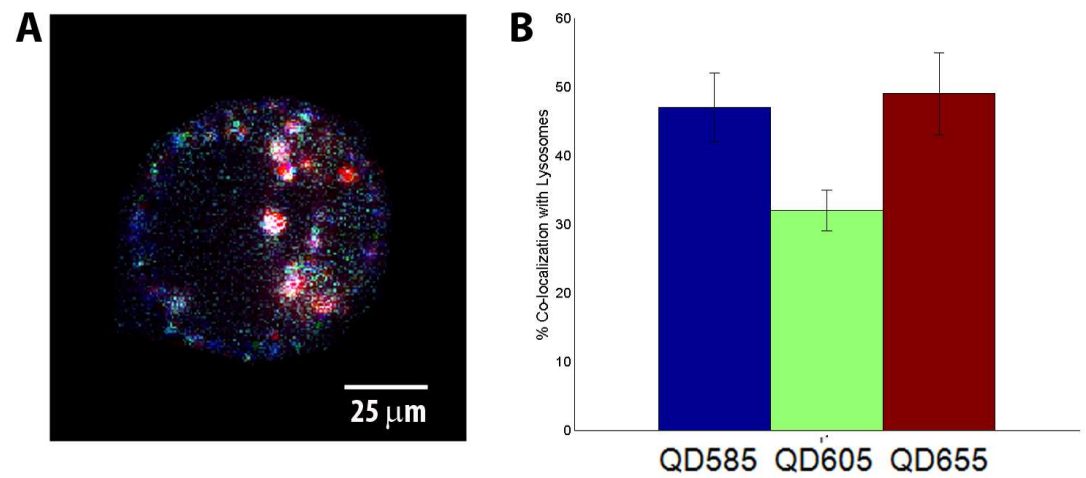

FIG. 2. Hyperspectral confocal microscopy allows simultaneous imaging of multiple fluorescent species in a single cell. (A). Pseudo-colored image of QD585 (blue), QD605 (green) and QD655 (red) in living RBL cell Treatment of cells with three quantum dot types, as well as a lysosomespecific dye permits quantitative evaluation of intra-cellular sorting for each QD. In (B), results show that the smaller and larger QD sizes (585 and 655 respectively) were preferentially sequestered in lysosomes, as compared to the intermediate sized particles (605). 